\title{
Resultados en el tratamiento quirúrgico de hernia discal lumbar, reporte a siete años de seguimiento
}

\author{
Results in the surgical treatment of lumbar disc hernia, \\ report of seven years follow up \\ Reyes-Sánchez A,* Reyes-Ayala C, ${ }^{\ddagger}$ García-Ramos CL, \\ Obil-Chavarría C, ${ }^{\ddagger}$ Alpizar-Aguirre A, ${ }^{\S}$ Rosales-Olivares $L^{\S}$
}

Instituto Nacional de Rehabilitación «Luis Guillermo Ibarra Ibarra».

RESUMEN. Introducción: La lumbalgia por hernia discal es provocada por el movimiento anormal intersomático, considerándose éste como factor etiológico de hernia discal, que en ocasiones es la indicación quirúrgica. Material y métodos: Con un diseño de estudio prospectivo, descriptivo, observacional y longitudinal en un período de Enero de 2000 a Diciembre de 2006. Muestra inicial de 195 pacientes, con 20 pacientes con criterios de inclusión a los siete años de seguimiento. Se tomaron en cuenta variables demográficas, dependientes e independientes. Se realizó análisis estadístico descriptivo comparando preoperatorio con la evolución a siete años. Resultados: Se englobaron los resultados en dos procedimientos: estabilización dinámica interespinosa y artroplastía, con 10 pacientes por cada procedimiento. Mediante la prueba de $\mathrm{T}$ y $\chi^{2}$ se observó significancia estadística al comparar los resultados de dolor y escala de Oswestry con parámetros de imagenología según Pfirrmann prequirúrgicos contra seguimiento final en los pacientes sometidos a estabilización dinámica. Para el grupo de artroplastía fue estadísticamente significativa la comparación de resultados de dolor con EVA (escala visual análoga) y función con escala de Oswestry, con una $\mathrm{p}<0.05$. Conclusión: Con este trabajo comprobamos que hubo significancia estadística al comparar los resultados clínicos de ambos procedimientos, observamos un porcentaje mínimo de complicaciones en los pacientes a quienes se les realizó estabilización dinámica en comparación con la artroplastía; por lo tanto, sugerimos realizar esta última
ABSTRACT. Introduction: Low back pain by herniated disc is caused by abnormal intersomatic movement, considering this as an etiological factor of disc herniation and the surgical indication. Material and methods: A prospective, descriptive, observational, longitudinal study design, in a period from January 2000 to December 2006. Initial sample of 195 patients, with inclusion criteria in 20 patients at seven years follow up. Demographic, dependent and independent variables were taken into account. Descriptive statistical analysis was conducted comparing preoperative with evolution to seven years. Results: Two groups were compared: dynamic interspinous stabilization and lumbar arthroplasty, with 10 patients for each procedure. Using $\mathrm{T}$ and $\chi^{2}$ test, statistical significance was observed when comparing the results of pain and Oswestry scale with parameters of imaging according to Pfirrmann pre surgical against final follow-up in patients undergoing dynamic stabilization. And for Arthroplasty was statistically meaningful comparison of results of pain with VAS (visual analogue scale) and function with Oswestry scale, with a p < 0.05. Conclusion: With this work we can see that there was statistical significance to compare clinical outcomes of both procedures, observing a minimum percentage of complications in patients who underwent dynamic stabilization compared with arthroplasty; therefore we suggest to perform the latter only in cases in all criteria, to

\section{Nivel de evidencia:}

* División Cirugía de Columna, Instituto Nacional de Rehabilitación «Luis Guillermo Ibarra Ibarra» (INRLGII) de la Secretaría de Salud (SS).

${ }^{\ddagger}$ Médico Ortopedista.

$\S$ Servicio de Cirugía de Columna, Instituto Nacional de Rehabilitación «Luis Guillermo Ibarra Ibarra» (INRLGII) de la Secretaría de Salud (SS).

Dirección para correspondencia:

Dr. Alejandro Reyes-Sánchez

Camino a Santa Teresa Núm. 1055-684, Col. Héroes de Padierna, Alcaldía Magdalena Contreras, CP. 10700, CDMX.

Tel.: 59991000, ext. 12209.

E-mail: alereyes@inr.gob.mx, areyes@vertebrae.com.mx

Este artículo puede ser consultado en versión completa en: www.medigraphic.com/actaortopedica 
sólo en casos en los que se reúnan adecuadamente todos los criterios para que los resultados clínicos y funcionales sean iguales a los esperados.

Palabras clave: Lumbalgia, hernia discal, tratamiento, estabilización, artroplastia, resultados.

\section{Introducción}

La hernia discal se define como el desplazamiento del núcleo, anillo o plataforma más allá de los márgenes del cuerpo vertebral adyacente. Como resultado del desplazamiento del disco existe una anormalidad en el margen de éste, en la mayoría de las ocasiones produce compresión en las raíces nerviosas, lo que da lugar a un cuadro clínico de lumbociática. ${ }^{1}$ El proceso patológico de la formación de una hernia discal obedece a una combinación de factores bioquímicos, degenerativos y mecánicos. ${ }^{2}$ La hernia de disco es un proceso gradual iniciado por fisuras del anillo fibroso causadas por problemas degenerativos con posible predisposición genética y es desencadenado en su fase final por una compresión aguda del disco, por lo general en flexión de columna. La flexión, o combinación de flexiónrotación-lateralización, puede ocurrir durante el levantamiento inadecuado de un objeto pesado o en un accidente. ${ }^{3}$ Otras veces hay un mecanismo de compresión axial, ${ }^{4}$ por tal motivo se considera una enfermedad discal más que un factor mecánico.

Las hernias de disco se consideran la causa más frecuente de lumbociática entre los 20 y 60 años de edad. ${ }^{5}$

La historia natural es por lo general benigna. En la mayoría de los casos un episodio agudo de ciática toma un curso breve. Esta fase por lo regular es seguida de un período subagudo o crónico de síntomas residuales. La mayoría de los pacientes se recuperan dentro de un mes, pero la tasa de recurrencia es de aproximadamente $10-15 \% .^{5}$ En la mayoría de los pacientes con extrusión o herniación secuestrada, los síntomas desaparecen con la herniación dentro de algunas semanas o meses sin importar si está contenida, extruida o migrada. ${ }^{6}$

Se han reportado estudios con tasa de satisfacción de $90 \%$ mediante administración de antiinflamatorios no esteroideos, reposo en cama, infiltración con esteroides y uso de corsés.?

Sin embargo, hay factores que predicen la falla en el tratamiento conservador como larga duración y la presencia de hernia secuestrada. ${ }^{4}$

En personas menores de 45 años, la lumbalgia es la principal causa de limitación de la actividad, en estudios realizados en nuestro país se ha comprobado que la lumbalgia por hernia discal es provocada por el movimiento anormal intersomático, considerándose éste como factor etiológico de hernia discal. ${ }^{8}$ La discectomía lumbar se ha vuelto el procedimiento de neurocirugía que se realiza más comúnmente meet adequately to be equal to the anticipated clinical and functional outcomes.

Keywords: Lumbalgia, disc hernia, treatment, stabilization, arthroplasty, results.

en EUA (Estados Unidos de América), con cerca de 300,000 procedimientos realizados cada año, debido al problema epidémico de lumbalgia esto excede un gasto de $\$ 50,000$ millones al año.

Aunque sólo 2\% de los pacientes con lumbalgia tienen una herniación aguda, un desproporcionado $30 \%$ de los costos anuales en EUA para el tratamiento de lumbalgia son gastados en ese porcentaje relativamente pequeño de pacientes. ${ }^{9}$

Los procedimientos de discectomía actuales no están dirigidos a tratar el daño del disco intervertebral y pueden inclusive agravar el daño ya existente. Por este motivo no es sorprendente que una descompresión neurológica exitosa con frecuencia es seguida por períodos de lumbalgia persistente, en referencia a pérdida de altura del disco y por consecuente alteración de la estabilidad vertebral, afectando la calidad de vida. ${ }^{10}$

En este momento carecemos de una casuística local donde se reporten los resultados con los tratamientos ofrecidos, por lo que se consideró conveniente hacer un estudio para comprobar con qué procedimiento se han obtenido mejores resultados clínicos en un lapso de cinco años.

Por lo tanto, nuestro objetivo general es identificar los resultados funcionales y de imagenología de los pacientes operados de hernia discal. A través de los objetivos específicos de describir las características demográficas de nuestra muestra, evaluar el dolor con la escala visual análoga (EVA), la incapacidad con la escala funcional Oswestry ${ }^{11}$ de los pacientes en el preoperatorio y al final del seguimiento medir espacio intervertebral en radiografías laterales preoperatorio y a los cinco años de seguimiento, describir cambios en el disco intervertebral en los casos en los que la técnica quirúrgica los conserva, con resonancia magnética establecer la condición preoperatoria y a los cinco años de seguimiento con escala Modic, valorar los cambios degenerativos en las plataformas vertebrales y los degenerativos discales en la clasificación de Pfirrmann modificada.

\section{Material y métodos}

Con un diseño de estudio prospectivo, descriptivo, observacional y longitudinal. Con muestreo abierto de los pacientes que se intervinieron en el Servicio de Cirugía de Columna por hernia discal lumbar. En un período de Enero de 2000 a Diciembre de 2006, con criterios de inclusión: edad mayor de 18 años, menor de 65 años de edad, género indistinto, expediente clínico y de imagen completo, con radiografías anteroposterior y lateral de columna lumbar, 
así como resonancia magnética preoperatorias y al final de su evolución.

Los criterios de eliminación al seguimiento fueron: deceso, desarrollo de enfermedad con afección a metabolismo óseo, pérdida de contacto del paciente o incongruencia de datos en el expediente clínico.

La muestra inicial fue de 195 pacientes, de los cuales sólo 20 cumplieron con los criterios de inclusión.

Se tomaron en cuenta variables demográficas de edad, sexo y ocupación. Las variables clínicas analizadas fueron el dolor y la incapacidad, valorados con la escala visual análoga y la escala funcional lumbar de Oswestry, respectivamente. La variable independiente fue la técnica quirúrgica (discectomía e instrumentación posterior o artroplastía). Se valoró la altura intervertebral en radiografía lateral y grado de degeneración Pfirrmann y Modic en resonancia magnética antes y después de la cirugía.

Se realizó estadística descriptiva de los datos mediante la estimación de frecuencias, porcentajes, media, mediana, rangos y desviación estándar. Se utilizó la prueba de comparación de medias para grupos relacionados, $\mathrm{T}$ pareada, cuando la distribución fue de tipo normal. La alternativa paramétrica que se usó fue la prueba de rangos con signo de Wilcoxon. Todas las pruebas se utilizaron considerando $\mathrm{p}<$ 0.05. Se usaron los paquetes estadísticos Excel y SPSS 20.

\section{Resultados}

A partir de la base de datos del Servicio de Cirugía de Columna de nuestro instituto, la cual constó al inicio del estudio de 195 pacientes, de los cuales sólo se incluyeron 20 , quienes reunieron de forma completa los criterios de inclusión, de ellos 13 hombres y siete mujeres.

Se dividieron en tres grupos por rangos de edad, de 30 a 39 años (40\%), de 40 a 49 (45\%) y de 50 a 59 (15\%). En 11 pacientes, de acuerdo a su estado civil se encontraban casados o en unión libre y nueve solteros.

La edad mínima fue de 30 años y la máxima de 56, con una media de 41.15, el peso varió entre 41 y $98 \mathrm{~kg}$, con una media de 72.57, la media de la talla fue de 1.67 con valores que variaron entre 1.52 y 1.78 y el promedio del índice de masa corporal fue de $25.98 \mathrm{~kg} / \mathrm{m}^{2}$.

Se realizó un seguimiento de siete años, se encontró que el nivel intervenido con más frecuencia fue L5-S1, seguido de L4-L5.

Los casos de este estudio se englobaron en dos procedimientos: estabilización dinámica interespinosa y artroplastía, con 10 pacientes por cada procedimiento. De los pacientes sometidos a artroplastía se detectó que el tipo de prótesis que se colocó con mayor frecuencia fue la PDN, con $20 \%$ del total de pacientes en el estudio y $40 \%$ del total de pacientes sometidos a este procedimiento, seguida de Prodisc con $15 \%$ del total y $30 \%$ de los casos correspondientes a artroplastía (Figura 1).

En cuanto a los resultados clínicos, de forma general, en ambos procedimientos se observó una mejoría de 5.45

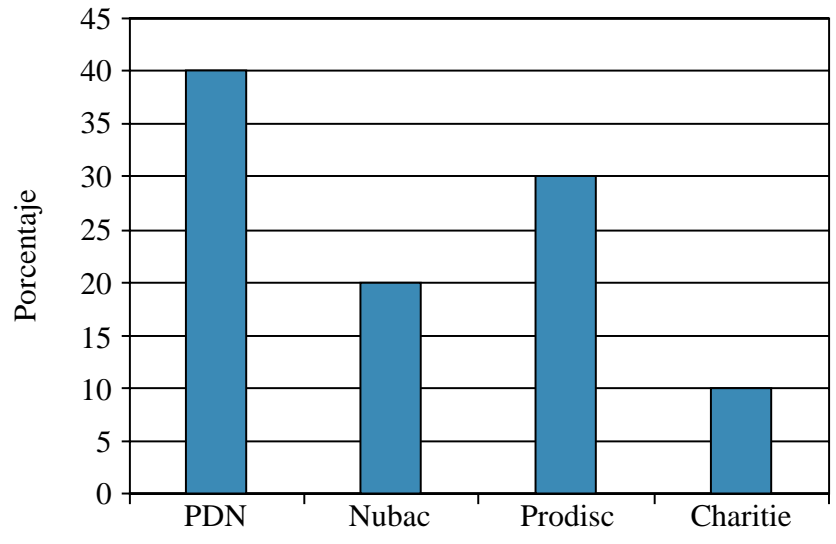

Figura 1: Frecuencia de los tipos de prótesis colocados en los pacientes del estudio.

puntos en escala visual análoga, con resultados que variaron desde cinco puntos hasta nueve en el prequirúrgico y de cero a seis en el postquirúrgico. Por grupos, se observó que para artroplastía la mejoría fue en promedio de 4.8, con valores que variaron desde cinco hasta nueve puntos en el prequirúrgico y desde uno hasta seis puntos en el postquirúrgico. Para la estabilización dinámica, notamos mejoría de 6.1 puntos en el prequirúrgico, variando desde cinco hasta nueve puntos y en el postquirúrgico de cero a cuatro puntos.

Al englobar ambos procedimientos, la calificación funcional con escala de Oswestry se observó mejoría de 34.6\% entre el prequirúrgico y el seguimiento a siete años. Previo a la cirugía se reportó que la mayoría de los pacientes tenían una escala de Oswestry categorizada con incapacidad severa (más de 60\%) siendo en el postquirúrgico lo que predominó como una mínima incapacidad por dolor lumbar.

En la artroplastía los resultados prequirúrgicos oscilaron entre 30 y $62 \%$ y al seguimiento final entre 6 y $42 \%$, con mejoría media de $32.6 \%$, con significancia estadística $(\mathrm{p}<$ 0.05) (Tabla 1).

En la estabilización dinámica, categóricamente en el prequirúrgico, se encontró a 70\% de los sujetos con una incapacidad severa, reportándose en el seguimiento final una mínima incapacidad por dolor lumbar en $80 \%$ de los pacientes (Tabla 1).

A nivel radiográfico, de forma general se observó disminución de la altura del espacio intersomático intervenido, en artroplastía hubo una disminución de 0.55 y $0.62 \mathrm{~mm}$ en estabilización dinámica, con una media de $0.575 \mathrm{~mm}$.

En resonancia magnética, en todos los pacientes se encontró con mayor frecuencia en el prequirúrgico un tipo II en escala de Modic en 45\%, seguido del tipo I con 35\%, predominando esta tendencia en el postquirúrgico con 55\% para el tipo II, seguido de $15 \%$ para el tipo I, se reportaron cuatro casos, que equivalen a $20 \%$, como no valorables debido a que en algunos pacientes sometidos a artroplastía no se preservó la integridad del disco. Para artroplastía en el prequirúrgico predominó el tipo II, con $40 \%$ de los casos, con un resultado similar a los siete años, disminuyendo sólo 
los casos que se encontraban en estadio I y III, siendo no valorables el mismo número de casos por lo ya comentado con anterioridad. En el caso de la estabilización dinámica, tanto en el prequirúrgico como en el seguimiento final, al igual que en artroplastía, predominó el tipo II de Modic, pero en 50 y $70 \%$, respectivamente.

Asimismo, de acuerdo a la escala de Pfirrmann, previo a la cirugía se reportaron 13 casos que ya se encontraban en estadio II, con $65 \%$ del total de los casos, se documentó en el postquirúrgico una frecuencia de 10 casos que se encontraron en estadio IV correspondiente a $50 \%$ e igualmente con cuatro casos, de los cuales no fue posible valorar el estadio por el tratamiento realizado, en este caso artroplastía. Por grupos estudiados, vimos que en artroplastía se detectaron seis casos que se encontraban en estadio III en el prequirúrgico (60\% de los casos), siendo en el seguimiento cuatro en estadio IV (40\%) y al igual que en la escala de Modic cuatro casos fueron no valorables. En la estabilización dinámica, en el prequirúrgico se observó que había siete pacientes en estadio III, equivalente a $70 \%$ del total de los pacientes sometidos a este tratamiento, para la valoración final notamos que la mayoría de los pacientes se encontraban en un estadio IV con seis pacientes (60\%) (Tabla 1).

Mediante la prueba de T y $\chi^{2}$ se observó que hubo significancia estadística al comparar los resultados de dolor con EVA y función con escala de Oswestry y parámetros de imagenología según Pfirrmann prequirúrgicos contra seguimiento final en los pacientes sometidos a estabilización dinámica (Tabla 1). Y para artroplastía fue estadísticamente significativa la comparación de resultados de dolor con EVA y función con escala de Oswestry, con una $\mathrm{p}<0.05$ (Tabla 2).

Por último, al comparar los resultados finales en ambos procedimientos de todas las variables, sólo se encontró significancia estadística en los resultados de dolor con EVA con una $\mathrm{p}<0.05$, no siendo así con el resto de las variables.

En cuanto a complicaciones, con la artroplastía se reportaron cuatro casos de migración del implante, por lo que fue necesario realizar el retiro de la prótesis en un segundo tiempo quirúrgico por las repercusiones clínicas que esto implicó en dos pacientes, los otros dos pacientes no manifestaron repercusiones clínicas de importancia para tomar en cuenta como criterio de retiro del implante. En la estabilización dinámica hubo un caso de lesión neurológica, siendo manejado mediante terapia física y rehabilitación, así como con el uso de una ortesis tobillo-pie para facilitar la marcha, sin afectación importante en este momento para la realización de actividades de la vida diaria.

\section{Discusión}

Este estudio representa un seguimiento a un plazo de siete años de los pacientes tratados de forma quirúrgica, en el que se incluyeron variables demográficas como edad, sexo y ocupación; dependientes de acuerdo al resultado funcional posterior al tratamiento quirúrgico valorado con sus respectivas escalas; e independientes como las técnicas quirúrgicas realizadas.

Fue evidente que las técnicas quirúrgicas que predominaron fueron la discectomía con ligamentoplastía (estabilización dinámica) y la artroplastía, por lo que en este estudio se englobaron los resultados en dichos grupos para hacer una comparación entre ambos.

Hoy en día, en la literatura no se ha descrito un estándar de oro para el tratamiento de hernia discal lumbar; sin embargo, debe considerarse que se han reportado buenos resultados con tratamiento conservador hasta de $87 \%$ con seguimiento de tres años, por lo que antes de pensar en realizar cirugía, ésta debe indicarse, si no existe una verdadera urgencia por dolor o con sintomatología neurológica. ${ }^{6}$

Cuando el tratamiento quirúrgico está indicado, la literatura describe una tasa de satisfacción de $80 \%$ en pacientes tratados quirúrgicamente contra $60 \%$ de quienes recibieron manejo conservador, manteniendo la relación hasta los cuatro años de seguimiento; pero a los 10 años la diferencia fue más significativa, al observar que $25 \%$ de los pacientes se sometieron a cirugía a partir de este tiempo, ${ }^{12}$ lo cual se ha percibido en la evolución que hemos tenido en nuestros pacientes.

En cuanto a la estabilización dinámica, Rosales-Olivares ${ }^{8}$ concluye que la ligamentoplastía interespinosa mejora la es-

\begin{tabular}{|c|c|c|c|c|c|c|c|c|}
\hline & \multicolumn{8}{|c|}{ Diferencias relacionadas } \\
\hline & \multirow[b]{2}{*}{ Media } & \multirow[b]{2}{*}{$\begin{array}{l}\text { Desviación } \\
\text { típ. }\end{array}$} & \multirow[b]{2}{*}{$\begin{array}{l}\text { Error típ. de } \\
\text { la media }\end{array}$} & \multicolumn{2}{|c|}{$\begin{array}{c}\mathrm{IC}_{95 \%} \\
\text { para la diferencia }\end{array}$} & \multirow[b]{2}{*}{$\mathrm{t}$} & \multirow[b]{2}{*}{ gl } & \multirow[b]{2}{*}{$\begin{array}{c}\text { Sig. } \\
\text { (bilateral) }\end{array}$} \\
\hline & & & & Inferior & Superior & & & \\
\hline EVA & 6.100 & 1.524 & 0.482 & 5.010 & 7.190 & 12.658 & 9 & 0.00001 \\
\hline Oswestry & 42.600 & 18.112 & 5.728 & 29.643 & 55.557 & 7.438 & 9 & 0.00001 \\
\hline Modic & -0.200 & 0.422 & 0.133 & -0.502 & 0.102 & -1.500 & 9 & 0.16800 \\
\hline Pfirrmann & -0.700 & 0.483 & 0.153 & -1.046 & -0.354 & -4.583 & 9 & 0.00100 \\
\hline $\begin{array}{l}\text { Altura del espacio } \\
\text { intersomático }\end{array}$ & 0.625 & 1.408 & 0.498 & -0.552 & 1.802 & 1.256 & 7 & 0.25000 \\
\hline
\end{tabular}




\begin{tabular}{|c|c|c|c|c|c|c|c|c|}
\hline & \multicolumn{5}{|c|}{ Diferencias relacionadas } & \multirow[b]{3}{*}{$\mathrm{t}$} & \multirow[b]{3}{*}{ gl } & \multirow[b]{3}{*}{$\begin{array}{l}\text { Sig. } \\
\text { (bilateral) }\end{array}$} \\
\hline & \multirow{2}{*}{\multicolumn{2}{|c|}{$\begin{array}{l}\text { Desviación } \\
\text { típ. }\end{array}$}} & \multirow[b]{2}{*}{$\begin{array}{l}\text { Error típ. } \\
\text { de la media }\end{array}$} & \multicolumn{2}{|c|}{$\begin{array}{c}\mathrm{IC}_{95 \%} \\
\text { para la diferencia }\end{array}$} & & & \\
\hline & & & & Inferior & Superior & & & \\
\hline EVA & 4.800 & 2.486 & 0.786 & 3.022 & 6.578 & 6.107 & 9 & 0.00001 \\
\hline Oswestry & 26.600 & 16.167 & 5.113 & 15.035 & 38.165 & 5.203 & 9 & 0.00100 \\
\hline Modic & -0.333 & 0.816 & 0.333 & -1.190 & 0.524 & -1.000 & 5 & 0.36300 \\
\hline Pfirrmann & -0.833 & 0.983 & 0.401 & -1.865 & 0.198 & -2.076 & 5 & 0.09300 \\
\hline $\begin{array}{l}\text { Altura del espacio } \\
\text { intersomático }\end{array}$ & 0.444 & 2.297 & 0.766 & -1.321 & 2.210 & 0.580 & 8 & 0.57800 \\
\hline
\end{tabular}

tabilidad segmentaria, permite la movilidad dentro de los rangos normales, conserva la altura discal a los cuatro años de seguimiento y ofrece mayor estabilidad con notable mejoría clínica, reportando mejoría de $80.3 \%$ en escala de Oswestry. Nuestro estudio reveló una mejoría de $42.6 \%$ en esta misma escala, con una $\mathrm{p}<0.05$, una mejoría del dolor en escala visual análoga de 6.1 puntos, lo cual comprueba una mejoría clínica estadísticamente significativa similar a la literatura; sin embargo, hay que considerar que existe un seguimiento mayor en comparación con los publicados.

Aunque no valoramos rangos de movilidad, radiográficamente se observó la disminución en promedio de $0.62 \mathrm{~mm}$ de la altura del espacio intersomático, lo que difiere con lo comentado en el estudio de Rosales-Olivares, ${ }^{8}$ sin olvidar nuestro tiempo de seguimiento.

En este estudio la mayoría de los pacientes antes de someterse al procedimiento de estabilización dinámica fueron valorados con escala Modic dentro del tipo II y I, y a pesar de que esta condición persistió en el postquirúrgico no hubo correlación con evolución clínica y funcional favorables como en lo descrito por Olcay Eser. ${ }^{13}$

Con el tema de artroplastía existe un reporte ${ }^{14}$ con un seguimiento a cuatro años, en el que hay una mejora en las condiciones clínicas y radiográficas de los pacientes así como aumento de la altura del espacio intersomático; refiere una mejoría de $35 \%$ en escala de Oswestry. Nosotros observamos en este estudio que hubo una mejoría de $32.6 \%$, siendo muy similar a lo reportado, pero con una disminución de la altura del espacio intersomático de $0.55 \mathrm{~mm}$. Creemos en la relación directa con tres años más de seguimiento.

En lo referente al aspecto de complicaciones, en un estudio realizado por Myung-Hoon Shin ${ }^{15}$ con una serie de 42 pacientes con 51 segmentos intervenidos con prótesis Pro Disc-L se demostró que el tropismo facetario mayor de $5^{\circ}$ es el principal predictor de artrosis facetaria. Chun-Kun Park $^{16}$ refiere que los cambios degenerativos de discos y facetas en su estudio fueron mínimos en un seguimiento de dos años usando la prótesis Prodisc II. Jiangbo Wei ${ }^{17}$ en un metaanálisis de ensayos controlados aleatorizados reveló que la artroplastía total de disco tiene una eficacia y seguridad significante comparable con la artrodesis a un seguimiento de dos años. Rosales-Olivares ${ }^{14}$ refiere en su estudio que la prótesis tuvo variaciones de acuerdo con su colocación en siete pacientes. En dos casos de migración se sobrepasaron los límites del cuerpo vertebral con repercusión fuera de clínica. En nuestro estudio se reportaron cuatro casos de migración protésica, de los cuales dos requirieron retiro del implante por invasión al conducto raquídeo, los otros dos casos sin repercusiones clínicas, similar a lo comentado por el autor.

A nivel de estabilización dinámica hay reportes de complicaciones, tales como lesiones de duramadre, lesiones de raíces nerviosas con sus consecuentes limitaciones motoras, recurrencia de hernia discal, ${ }^{18}$ similar a nuestro estudio, pero con un índice mucho menor que lo descrito en artroplastía.

Consideramos que una de las debilidades de este estudio es el bajo número de pacientes incluidos en la serie, a pesar de los registrados en la base de datos, siendo la causa principal la pérdida de contacto con el paciente, por lo que sugerimos en un futuro la realización de otro estudio, quizá con un seguimiento mayor, para incluir otras técnicas quirúrgicas y hacer una descripción más amplia de mejoría y de complicaciones en cada una de ellas.

\section{Conclusión}

Con este trabajo comprobamos que hubo significancia estadística al comparar los resultados clínicos de ambos procedimientos, también observamos un porcentaje mínimo de complicaciones en los pacientes a quienes se les realizó estabilización dinámica en comparación con la artroplastía; por lo tanto, sugerimos realizar esta última sólo en casos en los que se reúnan adecuadamente todos los criterios para que los resultados clínicos y funcionales sean iguales a los esperados.

\section{Bibliografía}

1. Froud R, Patterson S, Eldridge S, Seale C, Pincus T, Rajendran D, et al. A systematic review and meta-synthesis of the impact of low back pain on people's lives. BMC Musculoskelet Disord. 2014; 15(1): 50. 
2. Ito K, Creemers L. Mechanisms of intervertebral disk degeneration/ injury and pain: a review. Global Spine J. 2013; 3(3): 145-52.

3. Bron, JL. Repair, regenerative and supportive therapies of the annulus fibrosus: achievements and challenges. Eur Spine J. 2009; 18: 301-13.

4. Sutheerayongprasert C, Paiboonsirijit S, Kuansongtham V, Anuraklekha S, Hiranyasthiti N, Neti S. Factors predicting failure of conservative treatment in lumbar-disc herniation. J Med Assoc Thai. 2012; 95(5): 674-80.

5. Biering-Sorensen F, Thomsen C. Medical, social and occupational history as risk indicators for lowback trouble in a general population. Spine. 1986; 11: 720-5.

6. Saal JA, Saal JS. Nonoperative treatment of herniated lumbar intervertebral disc with radiculopathy: an outcome study. Spine. 1989; 14: 431-7.

7. Postacchini F. Management of herniation of the lumbar disc. $J$ Bone Joint Surg [Br]. 1999; 81-B: 567-76.

8. Rosales-Olivares LM, Alpízar-Aguirre A, Miramontes-Martínez V, Zárate-Kalfópulus B, Reyes-Sánchez A. Estabilización dinámica interespinosa en disectomía lumbar. Seguimiento de cuatro años. Cir Cir. 2010; 78: 495-9.

9. Porchet F. Microdiscectomy compared with standard discectomy: an old problem revisited with new outcome measures within the framework of a spine surgical registry. Eur Spine. J 2009; 18 (Suppl 3): S360-6.

10. Koebbe C. Maroon JC, Abla A, El-Kadi H, Bost J. Lumbar microdiscectomy: a historical perspective and current technical considerations. Neurosurg Focus. 2002; 13(2): E3.

11. Flórez-García MT, García-Pérez F, Alcántara-Bumbiedro S, EchávarriPérez C, Urraca Gesto A, Alañón- Caballero J, et al. Diseño y desarrollo de una aplicación informática para la escala de incapacidad por dolor lumbar de Oswestry. Patología del Aparato Locomotor. 2005; 3(2): 120-31.

12. Postacchini F, Postacchini R. Operative management of lumbar disc herniation: the evolution of knowledge and surgical techniques in the last century. Acta Neurochir Suppl. 2011; 108: 17-21.

13. Olcay E, Cengiz G, Mehdi S, Tunc O, Ahmet LA, Yaprak A, et al. Dynamic stabilisation in the treatment of degenerative disc disease with Modic changes. Adv Orthop. 2013; Adv Orthop. 2013; 2013: 806267. http://dx.doi.org/10.1155/2013/806267.

14. Rosales-Olivares LM, Alpízar-Aguirre A, Miramontes-Martínez V, Zárate-Kalfópulus B, Reyes-Sánchez A. Dynamic interspinous stabilization in lumbar discectomy: 4-year follow-up. Cir Cir. 2010; 78(6): 492-6.

15. Shin MH, Ryu KS, Hur JW, Kim JS, Park CK. Association of facet tropism and progressive facet arthrosis after lumbar total disc replacement using ProDisc-L $\left.{ }^{(}\right)$. 2013; 22(8): 1717-22.

16. Park CK, Ryu KS, Jee WH. Degenerative changes of discs and facet joints in lumbar total disc replacement using ProDisc II minimum two-year follow-up. Spine. 2008; 33(16): 1755-61.

17. Jiangbo Wei, Yueming Song, Lin Sun, Chaoliang LV. Comparison of artificial total disc replacement versus fusion for lumbar degenerative disc disease: a meta-analysis of randomized controlled trials. Int Orthop. 2013; 37(7): 1315-25.

18. Xu L, Yu X, Bi LY, Liu GZ, Li PY, Qu Y, et al. Intermediate and longterm follow-up evaluation of posterior dynamic lumbar stabilization in lumbar degenerative disease. Zhonghua Wai Ke Za Zhi. 2012; 50(9): 792-6. 